\title{
CORRIGENDUM: A NEW EXPERIMENTAL APPROACH TO MACH'S PRINCIPLE AND RELATIVISTIC GRAVITATION
}

\section{James F. Woodward}

In the above-mentioned paper, which appeared in Found. Phys. 3, 497-506 (1990), the following corrections should be made:

1. In the title "GRAVIATION" should read "GRAVITATION".

2. In line 14 of page 500 "to" should be "To".

3. In Eq. (7) " $\nabla \cdot \boldsymbol{a}$ " should be " $\nabla \cdot \boldsymbol{A}$ ".

4. In Eqs. (9) and (10) " $4 \pi G \rho$ " should be " $-4 \pi G \rho$ ".

5. In the sentence leading to Eq. (11) " $\Phi_{c} / c^{2}=\beta$ " should be " $\Phi_{c} / c^{2} \rho=\beta "$.

6. In lines 10 and 12 on p. 504 "plastic capacitors" should be "Plastic Capacitors."

7. For "event horizon" everywhere read "particle horizon." 